\title{
Alternative Procedure for One-Stage Nostril Reconstruction: A Case Report
}

\author{
Iswinarno D. Saputro ${ }^{1}$, Heri Noviana ${ }^{2}$ \\ ${ }^{1}$ Plastic, Reconstructive, and Aesthetic Specialist Consultant, Airlangga University / DR. Soetomo Teaching \\ Hospital, Surabaya, Indonesia \\ ${ }^{2}$ Resident of Plastic, Reconstructive, and Aesthetic Surgery Department, Airlangga University / DR. Soetomo \\ Teaching Hospital, Surabaya, Indonesia \\ iswinarno.doso@fk.unair.ac.id
}

\begin{abstract}
Closure of nasal defect remains a challenge for surgeons. There are several ways to do nasal reconstruction, including donor site for nasal reconstruction due to its vascularity that is superior to other areas. Methods: This is a case report of a 75-year old male patient with Squamous Cell Carcinoma on his left nostril who underwent wide excision. This action left a 4-cm defect, whilst the nasal septum remained intact. This defect closure was using $V-Y$ advancement full thickness nasolabial flap in one step. This defect closure was using $V-Y$ advancement full thickness nasolabial flap in one step. Results: The defect closure was performed with tum over local (nasobialis) flap from the left side of the nasal cartilage. The superiority of this flap, it is able to be performed bulking, so that closure with turn over local nasolabial flap has been a sole option in nostril reconstruction for decades, which gave very good cosmetic results. After being followed for 1 month after surgery, the flap was viable, the contour was well formed, the scar was minimal, and there was no respiratory disruption. The patient was satisfied by the results. Conclusion: Nasolabial turn over local flap can be used as an option to close a relatively wide nostril
\end{abstract}

Keywords: turn over local flap; nostril defect; post wide excision; local facial flap; nasolabial flap

\section{Introduction}

Squamous Cell Carcinoma of the face and neck is quite common, due to direct exposure to ultraviolet rays [1][7]. The mainstay of treatment for these malignancies is wide excision and usually difficult to do primary suture, especially in nasal region with its unique shape deeply attached to the nasal cartilage, makes it more complicated to do the reconstruction [2][6]. Nostril consists of 4 layers, the most outer layer is skin, then sequentially, inner nasal layer, cartilage, and fibrous fatty tissue [6]. There are several ways to perform defect closure on nostrils, including forehead flap and nasolabial flap. In fact, nasolabial is an ideal donor site for nasal reconstruction due to its vascularity that is superior to other areas. Although the nasolabial flap is an old method, it still often being used as it has superiorities such as; being done in one stage, possibly being done under local anesthesia, being performed quite easily, requiring relatively short duration of surgery, providing a full thickness donor, and minimal scarring [2][4].

\section{Research Methods}

A 75-year-old male patient complaint about Squamous Cell Carcinoma on his left nostril with $4 \mathrm{~cm}$ diameter was performed wide excision including a $6 \mathrm{~mm}$ safety margin at DR. Soetomo Teaching Hospital, Surabaya, and East Java, Indonesia. This action left a defect on the left nostril, approximately $5 \mathrm{~cm}$ in diameter, which did not pass through the 
nasal septum. It remained intact, so V-Y advancement nasolabial flap was the most suitable choice to cover the defect. This defect closure was performed in one step simultaneously after wide excision under general anesthesia for patient's comfort. The incision design was drawn by drawing 2 lines along $10 \mathrm{~cm}$ length starting from the most superior border of the defect and the second line starting from the inferior border of the defect which met at the lateral border of the left nasolabial sulcus by both of those lines forming a 300 degrees angle. The incisions were made according to that design. The flap was taken full thickness, by which reached the buccal mucosa. Then the flap was pulled towards the defect, the donor area was treated with primary suture. All area was sutured by interrupted sub dermal sutures with 5-0 absorbable and interrupted sutures with 6-0 nonabsorbable monofilament for the skin. Duration of surgery was 40 minutes.

Postoperatively, the flap was evaluated for changes in color, viability, and capillary refill time periodically. The patient was given antibiotic and analgesic therapy, and was getting wound toilet every 3 days with modern dressings. The patient was discharged on the third day postoperative. The flap was viable, no signs of necrotic and infection. Furthermore, the skin sutures were removed on day 7. After 2 weeks postoperatively, the flap remained viable, minimal scarring, minimal bulking, and no surgical wound infection. Patients were advised to avoid sun exposure for better scarring.

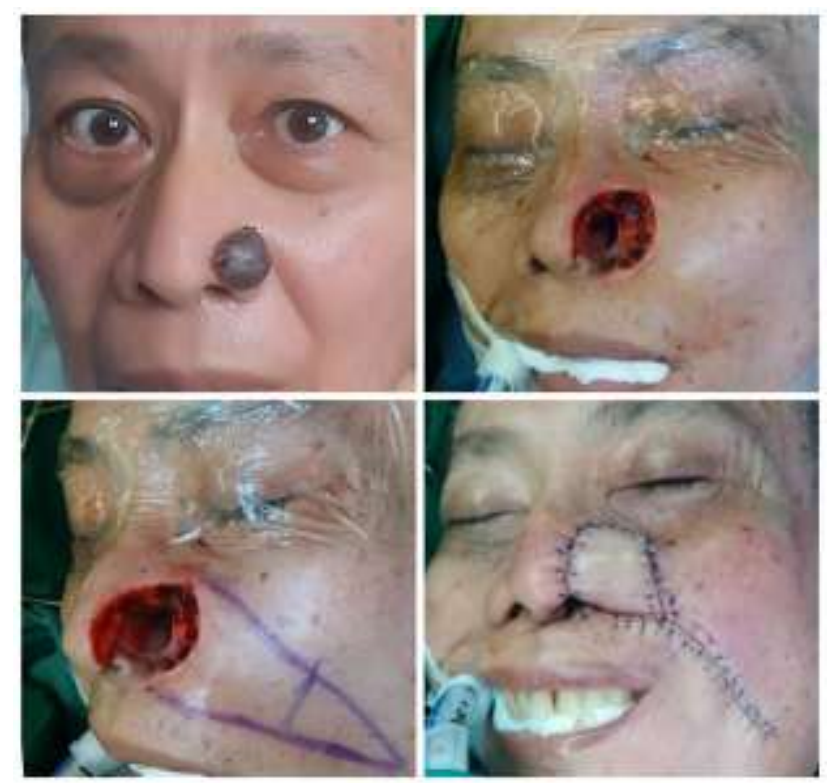

Figure 1. Clinical Picture of the Patient (a) Pre-surgery (b)Post-wide excision defect (c) $V-Y$ Advancement (local) Nasolabial Flap Design (d) Post-covering Defect with Full Thickness Nasolabial (local) Flap

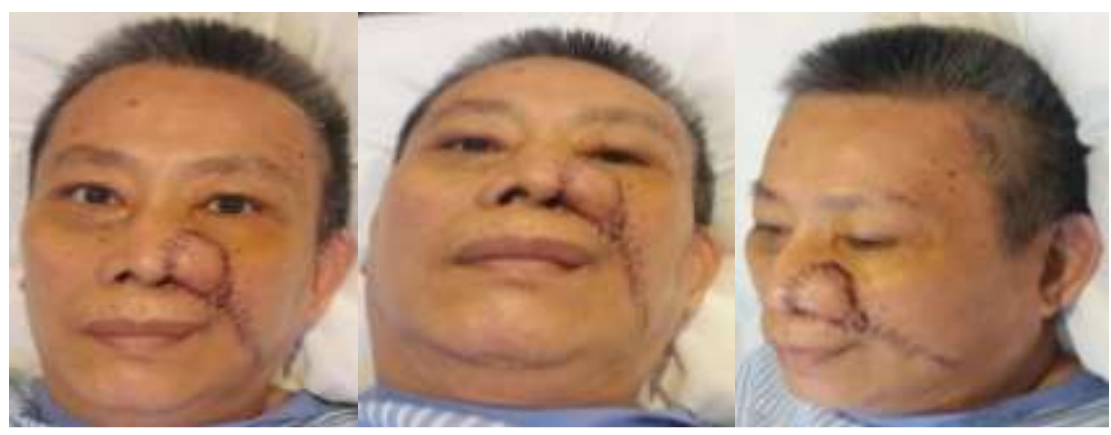

Figure 2. Clinical Postoperative Picture. Flap was Viable, No Active Bleeding, No Hematoma, Good Healing 


\section{Discussion}

Facial reconstructive surgery has a wide variety of options with their advantages and disadvantages. To choose a reconstruction for nasal defects, especially for nostrils, is a challenge for surgeons. To appear normal, it must have the proper dimension, volume, position, projection, symmetry and it is a best choice using autogenous tissue [9]. In this case study, the authors chose a turn-over local (nasolabial) flap from left side to close the defect on left nostril. Of course, the choice of reconstruction must still be in accordance with the standard choice of wound closure; primary closure is preferred, then skin graft or the flap can be selected if primary or graft closure is not possible. However, the defect structure in this case and its location on nostril was not able to be closed primarily. Selection of a full thickness skin graft in such a case could give a prominent scar, inappropriate skin contour and colour, requiring further reconstruction [6][7].

Nasiolabial flap itself is divided into 5 kinds depending on the composition; skinless flap, epidermis-dermis flap, epidermis-dermis-subcutaneous flap, myocutaneous flap, full thickness flap (which consists of skin to buccal mucosa). Its selection can be adjusted to the defect that needs to be patched. In terms of selecting the artery that is the main supply of this flap, several options can be made; the facial artery located on the inferior part of the flap, the angular artery located on the superior part of the flap, the lateral nasal branch of the facial artery located in the medial part of the flap, and the infraorbital artery (branch of the maxillary artery). This selection is based on the findings at surgery that can be identified in advance during the operation using Doppler ultrasound. Based on the transfer direction, the nasolabial flap is divided into VY advancement flap, rotation flap which is the main choice for reconstruction on upper lip area, transposition flap, and tunnelized flap which usually being selected for the defect area relatively far from the donor so that the donor area needs to be undermining to minimize distance between donor and recipient [4].

In this case, a full thickness V-Y advancement nasolabial flap was selected according to the defect. Turn over local (nasolabialis) flap can be performed under local anesthesia, can be done in one stage, high vascularization, minimal bulking so that closure of the wound defect with this kind of flap has been the method of choice in nostril reconstruction for decades, which was cosmetically excellent due to the same skin tone and minimal donor site morbidity [2][3][9]. In this case, the operator performed a wide excision with 6-mm safety margin due to the tumor was larger than $2 \mathrm{~cm}$ [5]. There was no studies stated about maximum defect size possible to be closed using this method, however, one study stated that the donor area with a maximum width of $5 \mathrm{~cm}$ was possible to be closed primarily by firstly undermining the donor site [3].

The complications of nasolabial flaps include temporary flap congestion, bulking, alar retrusion, and flap necrosis. However, with good surgical technique, well precise thickness of donor flap, minimization of injury to the vascular tissue around the flap are key to minimize the risk of those complications. In a study conducted by Bilal et al. in 2014 on 56 patients who underwent nostril reconstructions with similar method, these complications were minimal [3]. Last but not least, esthetic is also great importance in performing nasal reconstruction [7].

After being followed for 1 month after surgery, the flap remained viable, the contour was well formed and looked symmetrical, acceptable scarring and bulking, also there was no disruption in breathing, so that the patient was quite satisfied with the results of his nostril reconstruction. 


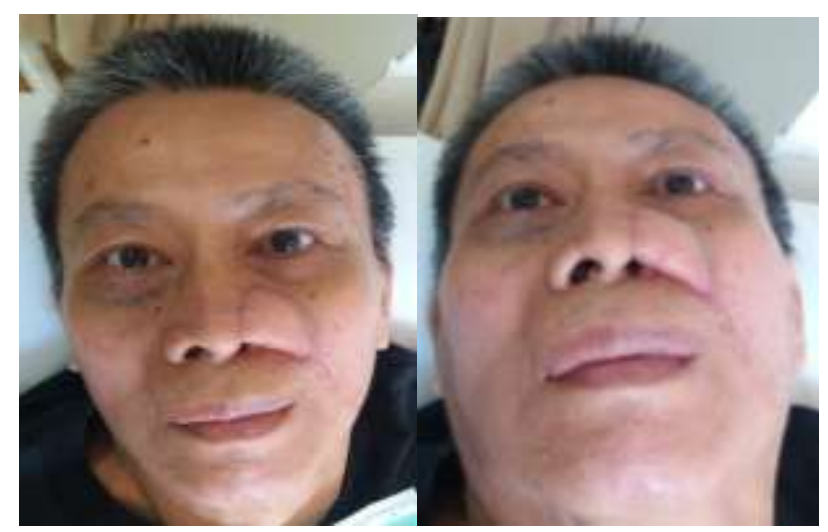

Figure 3. Clinical Picture of the Patient 1 Month after Surgery. Flap Remained Viable, Good Wound Healing, Acceptable Scarring and Bulking

\section{Conclusion}

Nasolabial turn over local flap can be used as an option to close a relatively wide nostril defects. It has same skin colour, good vascularity, acceptable scarring and bulking, satisfying aesthetic value, and does not require very high skills to perform it. With good surgical technique, well planned flap design, and minimization of injury to surrounding tissues, complications such as alar retrusion, flap necrosis, and flap congestion are possible to be avoided.

\section{References}

1. Jung HC, Young JK, Hoon K, Sang HN, Young WC. Distribution of basal cell carcinoma and squamous cell carcinoma by facial esthetic unit. Arch Plast Surg. 2013;40(4):387-391.

2. Aksam E, Aksam B, Karaaslan O, Durgun M. Nasolabial flaps for nasal reconstruction: pros and cons. Turk Journ of Plas Surg. 2018;26(4):151-155.

3. Bilal M, Ullah I, Ehsanullah. Nasolabial flap: a workhorse for reconstruction of nasal ala after tumor resection. JKCD. 2014;5(1):11-15.

4. Rahpeyma A, Khajehahmadi S. The place of nasolabial flap in orofacial reconstruction : a review. Ann Med Surg. 2016;12:79-87.

5. Najjar T, Meyers AD, Monroe MM, Alam M, Balbak LM, Caputy G, de la Torre JI, DeBacker C, Dryden RM, Duffy MT, Elston DM, et al. What are the recommended margin sizes in surgical excision of cutaneous squamous cell carcinoma (cSCC)? Available at https:/www.medscape.com/answers/1965430-85459/what-are-therecommended-margin-sizes-in-surgical-excision-of-cutaneous-squamous-cellcarcinoma-cscc [Internet] [cited on 24th April 2021]

6. Young JP, Gyu HK, Jun OK, Woo SR, Kyung SL. Reconstruction of nasal ala and tip following skin cancer resection. Arch Craniofac Surg. 2019;20(6):382-387.

7. Kaya I, Uslu M, Apaydin F. Defect reconstruction of the nose after surgery for nonmelanoma skin cancer: our clinical experience. Turk Arch Otorhinolaryngol. 2017;55(3):111-118.

8. Thorne CH, Chung KC, Gosain AK, Gurtner GC, Mehrara BJ, Rubin JP, Spear SL. Grabb and Smith's plastic surgery seventh edition. Philadelphia : Lippincott Williams \& Wilkins. 2014;4(33):361-371

9. Mathes SJ, Hentz VC. Mathes plastic surgery second edition. Philadelphia : Saunders/Elsevier. 2005., 